\title{
EFECTO DE DIETAS EN ADULTOS DE Spodoptera cosmioides (LEPIDOPTERA: NOCTUIDAE) SOBRE LA FERTILIDAD, FECUNDIDAD Y LONGEVIDAD DEL ADULTO
}

\author{
Curis, M. C. ${ }^{1 ;}$ BertolaccinI, I. ${ }^{1} \&$ LUTZ, A. ${ }^{1}$
}

\begin{abstract}
RESUMEN
La aparición de cultivares de soja Bt + RR2Y determinó la emergencia de una nueva plaga, Spodoptera cosmioides, una plaga altamente polífaga de amplia distribución. La cría de insectos en cámaras de cría es necesaria para estudios de investigación básica y aplicada. El objetivo del trabajo fue evaluar el efecto de tres dietas ricas en carbohidratos (solución de miel, de jarabe de glucosa y de jarabe de maíz) en la alimentación de los adultos y determinar su efecto sobre la fecundidad, fertilidad y supervivencia de $S$. cosmioides. Se determinó que, si bien no se hallaron diferencias entre los tratamientos, la composición de los azúcares de las dietas, puede afectar los parámetros reproductivos de esta plaga, ya que las hembras alimentadas con solución de miel natural, más rica en compuestos orgánicos, aumentaron la fecundidad, reflejado en una mayor puesta y eclosión de huevos y la longevidad de los adultos.
\end{abstract}

Palabras clave: cría artificial, carbohidratos, dietas de adultos.

\begin{abstract}
The effect of diet in adult stages of Spodoptera cosmioides (Lepidoptera: noctuidae) on fertility, fecundity and adult longevity.

The occurrence of the Bt + RR2Y soybean cultivars determined the appearance of a new pest, Spodoptera cosmioides, a highly polyphagous, widely distributed pest. Insect breeding in chambers is necessary for basic and applied research. The aim of the work was to evaluate the effect of three diets rich in carbohydrates (honey solution, glucose syrup and corn syrup) in the diet of adults and
\end{abstract}

1.- Departamento de Producción Vegetal. Facultad de Ciencias Agrarias (UNL). (3080) Esperanza, provincia de Santa Fe. Email: mcuris@fca.unl.edu.ar

Manuscrito recibido el 6 de septiembre de 2017 y aceptado para su publicación el 7 de noviembre de 2017. 


\section{C. Curis et al.}

to determine their effect on fertility, fecundity and survival of $S$. cosmioides. It was determined that, although no differences were found between the treatments, the composition of the diets sugars can affect the reproductive parameters of this pest, since the females fed with solution of natural honey, rich in organic compounds, increased fertility, reflected in greater egg laying and hatching and longevity of adults.

Key words: artificial breeding, carbohydrates, adult diets.

\section{INTRODUCCIÓN}

En Argentina, en las últimas campañas agrícolas se detectó la presencia de Spodoptera cosmioides en cultivares de soja $\mathrm{Bt}+\mathrm{RR} 2 \mathrm{Y}$, en densidades poblacionales elevadas, tanto en estado vegetativo como reproductivo del cultivo (1). Esta especie es muy polífaga (2) y más voraz que otras, causando una elevada defoliación y consumo de estructuras reproductivas de la soja (3). Se distribuye preferentemente en regiones cálidas, desde Panamá hasta el centro de Argentina, en las provincias de Chaco, Tucumán, Santa Fe, Entre Ríos y Buenos Aires (2).En la mayoría de los insectos, los nutrientes adquiridos en la etapa larval juegan un papel importante al momento de la reproducción de los mismos. En el orden Lepidoptera depende también de los nutrientes obtenidos en la etapa adulta, donde la ingesta de carbohidratos y aminoácidos pueden tener efectos en la reproducción $(4,5)$, debido a que influyen en el número de posturas, el número de huevos por postura (6), el número de huevos eclosionados y la vida media del adulto $(7,8)$.

En la cría de insectos en laboratorio, el uso de dietas artificiales, provee una fuente de alimento fácil de manejar, eliminándose el problema de trabajar con la planta huésped o con parte de la misma y evita los riesgos de contaminación con entomopatógenos. Es indispensable que los insectos cuenten con los elementos necesarios para su normal desarrollo para mantener experimentalmente poblaciones, al momento de realizar investigaciones, logrando, en lo posible, niveles de fertilidad y fecundidad similares a los que ocurren en condiciones de campo. Diversos trabajos confirman la existencia de una relación directa entre la clase de alimento y los parámetros biológicos, tales como el crecimiento y desarrollo de especies del género Spodoptera $(9,10,11,12,13,14)$.

La miel es el principal alimento de muchos insectos adultos. Producido por las abejas a partir del néctar de las flores, los carbohidratos, son el componente primordial, representado por fructosa y glucosa (38\% y 31\%, en promedio respectivamente) y representan el $85 \%$ de los sólidos, el resto incluye al menos otros 25 azúcares complejos, algunos en niveles muy bajos. Contiene además, aproximadamente 0,5\% de proteínas, entre 11 y 21 aminoácidos libres y baja cantidad de vitaminas. Los minerales son variables $(0,02$ a $1,0 \%)$, el $\mathrm{K}+$ cerca de la tercera parte y excede en 10 veces al $\mathrm{Na}+, \mathrm{Ca}++$ y $\mathrm{Mg}++$ y menos abundantes son: $\mathrm{Fe}+++, \mathrm{Mn}++, \mathrm{P}+++; \mathrm{S}++\mathrm{y}$ $\mathrm{Si}++++$ (15). Contiene además ácidos orgánicos y residuos de polen (16). El jarabe de maíz (fructosa) se obtiene comercialmente a partir del almidón de maíz, es un polisa- 